\title{
Long term use of donepezil and QTc prolongation
}

\author{
Jason Kho ${ }^{1}$, Adam Ioannou ${ }^{2}$, Amit Mandal ${ }^{1}$, Andrew Cox ${ }^{1}$, Ashraf Nasim ${ }^{1}$, Sofia Metaxa ${ }^{1}$, \\ and Constantinos Missouris ${ }^{1}$ \\ ${ }^{1}$ Wexham Park Hospital \\ ${ }^{2}$ Royal Free London NHS Foundation Trust
}

May 6, 2020

\begin{abstract}
Background and Purpose: The neurocognitive benefits of donepezil are well recognised, but the potential side effects on cardiac conduction remain unclear. Our objectives are to investigate whether long-term donepezil therapy is associated with electrocardiographic (ECG) changes and in particular to assess its effects on the QT interval. Experimental approach: We conducted a single centre retrospective analysis of patients admitted to our institution on donepezil therapy over a 12-month period. 59 patients were identified as suitable for analysis. An admission resting 12-lead ECG was obtained and compared to their ECG prior to commencement of donepezil therapy to assess for any significant difference in ECG parameters. Key Results: Donepezil significantly prolonged the PR $(\mathrm{P}=0.04)$, QRS $(\mathrm{P}=0.04)$ and $\mathrm{QT}(\mathrm{P}=0.002)$ intervals. The increase in $\mathrm{QT}$ intervals remained significant on correction for heart rate; resulting in 8 (13.6\%) patients developing high arrhythmogenic risk based on assessment using QT nomogram plots. Concomitant use of tricyclic antidepressants was associated with significant QT prolongation, while use of rate limiting calcium channel blockers was associated with significant PR prolongation, and beta-blockers with reduction in heart rate. Conclusion and Implications: Our results clearly demonstrate that long-term use of donepezil results in prolongation of the QT interval. We suggest ECG evaluation should take place before and after donepezil initiation, and clinicians should be even more vigilant in those prescribed tricyclic antidepressants.
\end{abstract}

\section{Long term use of donepezil and QTc prolongation}

Jason $\mathrm{Kho}^{1}$, Adam Ioannou ${ }^{2}$, Amit K J Mandal ${ }^{1}$, Andrew Cox $^{1}$, Ashraf Nasim ${ }^{1}$, Sofia Metaxa ${ }^{1}$, Constantinos G Missouris ${ }^{1,3}$

${ }^{1}$ Wexham Park Hospital, Frimley Health NHS Foundation Trust, UK

${ }^{2}$ Royal Free Hospital, Royal Free London NHS Foundation Trust, UK

${ }^{3}$ University of Cyprus Medical School, Nicosia, Cyprus

Corresponding Author

Prof Constantinos G Missouris, MD, FRCP

Department of Cardiology and Internal Medicine

Wexham Park Hospital

Frimley health NHS Foundation Trust

Wexham Street, Slough UK

Telephone: 01753633000

E-mail: dinos.missouris@nhs.net 
Abstract: 221 words

Manuscript: 2532 words

Funding: The authors received no specific funding for this work.

Disclosures : No potential conflict of interest was reported by the authors.

Author Contributions : All authors confirm that they contributed to the design and implementation of the research, to the analysis of the results and to the writing of the manuscript.

Acknowledgements: This work was aided and supported by the Pharmacy Department of Wexham Park Hospital, Frimley Health NHS Foundation Trust, United Kingdom.

Abstract

\section{Background and Purpose:}

The neurocognitive benefits of donepezil are well recognised, but the potential side effects on cardiac conduction remain unclear. Our objectives are to investigate whether long-term donepezil therapy is associated with electrocardiographic (ECG) changes and in particular to assess its effects on the QT interval.

\section{Experimental approach:}

We conducted a single centre retrospective analysis of patients admitted to our institution on donepezil therapy over a 12-month period. 59 patients were identified as suitable for analysis. An admission resting 12-lead ECG was obtained and compared to their ECG prior to commencement of donepezil therapy to assess for any significant difference in ECG parameters.

\section{Key Results:}

Donepezil significantly prolonged the $\mathrm{PR}(\mathrm{P}=0.04)$, $\mathrm{QRS}(\mathrm{P}=0.04)$ and $\mathrm{QT}(\mathrm{P}=0.002)$ intervals. The increase in QT intervals remained significant on correction for heart rate; resulting in 8 (13.6\%) patients developing high arrhythmogenic risk based on assessment using QT nomogram plots. Concomitant use of tricyclic antidepressants was associated with significant QT prolongation, while use of rate limiting calcium channel blockers was associated with significant PR prolongation, and beta-blockers with reduction in heart rate.

\section{Conclusion and Implications:}

Our results clearly demonstrate that long-term use of donepezil results in prolongation of the QT interval. We suggest ECG evaluation should take place before and after donepezil initiation, and clinicians should be even more vigilant in those prescribed tricyclic antidepressants.

Keywords: Cardiac pharmacology, pharmacodynamics, ageing/aging, donepezil, electrocardiography, cardiac arrhythmias, cholinesterase inhibitors

\section{Introduction}

Donepezil is an acetylcholinesterase inhibitor primarily used to treat mild to moderate Alzheimer's dementia (AD). While the neurocognitive benefits of donepezil are well documented, its adverse affects on cardiac conduction remain unclear. Several case studies have reported QT prolongation and subsequent Torsades de Pointes (TdP) associated with donepezil use in patients with AD (Kitt \& Irons et al., 2015; Mandal \& Khan et al., 2019; Malik \& Hamid et al., 2019; Takaya \& Okamoto et al., 2009) while others have reported bradycardia and PR prolongation, resulting in subsequent medication cessation (Bordier \& Garrigue et al., 2003; Hernandez \& Farwell et al., 2009). Smaller scale studies to date have shown that donepezil is relatively safe to use in the older population. Common parasympathetic side-effects have been associated with its use, but importantly these small-scale observational studies have not reported any sinister cardiac conduction abnormalities such as significant QT prolongation, subsequent arrhythmia or syncopal episodes (Bordier \& Garrigue et al., 2006; Igeta \& Suzuki et al., 2014; Wang \& Wu et al., 2018). 
The British National Formulary (BNF, 2020) describes bradycardia as an uncommon side-effect and cardiac conduction disorders to be a rare, or very rare side effect of donepezil. First-degree atrioventricular (AV) block and supraventricular tachycardia were reported to be infrequent side-effects by the United States Food and Drug Administration (FDA, 2020). Physicians are advised to practise caution when prescribing donepezil in patients with cardiac conduction disorders, but despite these warnings there remains on-going debate as to whether a resting 12-lead electrocardiogram (ECG) is required prior to initiation of donepezil therapy (Rowland \& Rigby et al., 2007).

The primary objective of our study is to investigate whether ECG changes are associated with donepezil therapy and in particular assess its effects on the QT interval. We also aim to evaluate whether gender, treatment duration, donepezil dose or concomitant use of medications are associated with conduction abnormalities and QT prolongation in patients prescribed donepezil therapy.

\section{Methods}

We carried out a retrospective analysis of all acute admissions of patients on donepezil treatment to our institution between March 2019 - March 2020. An admission resting 12-lead ECG was obtained and compared to their ECG prior to commencement of donepezil therapy. All data were collected from our local hospital electronic care record and memory clinic database. Patient characteristics and demographics were recorded, including indications for donepezil, comorbidities and left ventricular function (assessed using the Simpson's biplane method on images obtained from a recent transthoracic echocardiogram). Baseline serum electrolytes (magnesium, adjusted calcium and potassium) and thyroid status were noted. Medications with potential side-effects on cardiac conduction or known to cause electrolyte disturbances, including betablockers, levothyroxine, rate-limiting calcium-channel blockers, diuretics, digoxin, tricyclic antidepressants, antipsychotics and selective serotonin reuptake inhibitor (SSRI)/selective serotonin noradrenaline reuptake inhibitor (SNRI) antidepressants were all recorded. In addition, the duration of donepezil therapy was established and rounded off to the nearest year.

Exclusion criteria included recent initiation of donepezil therapy ( $<6$ months), patients with electrolyte disturbances or deranged thyroid function on admission, patients with a paced rhythm on ECG and those patients in whom an ECG prior to commencement of therapy could not be obtained.

\section{Measuring Electrocardiographic Parameters}

All patients underwent a 12-lead resting ECG using a $25 \mathrm{~mm} /$ second paper speed and standardised at $0.1 \mathrm{mV} / \mathrm{mm}$. The patient's heart rhythm was analysed by a cardiology registrar or consultant. Heart rate (HR), PR and QRS intervals were calculated automatically by the ECG apparatus. The QT interval was measured from multiple leads (chest leads V3-V6 and lead II) and the median QT was calculated. Due to the discrepancies in formulae used for correcting QT intervals; all four commonly used formulae (Bazett, Fredericia, Framingham and Hodges) were included. The QT intervals were plotted on a QT nomogram (a plot of uncorrected QT interval against heart rate) to predict arrhythmogenic risk (Chan \& Isbister et al., 2007).

\section{Ethics}

This survey was approved by the trust audit department with reference CB582. As a registry report using clinically collected, non-identifiable data, this work does not fall under the remit of National Health Service Research Ethics Committees.

\section{Data and Statistical Analysis}

Data was analysed with SPSS Version 26 software. Continuous variables were expressed as mean \pm standard deviation and categorical variables were summarised as frequencies and percentages. Normality of all continuous variables was tested with the Kolmogorov-Smirnov test. Since the variables were not normally distributed, the Wilcoxon signed rank test was used to compare the mean in all ECG parameters while the Mann-Whitney U test was used to compare the changes in ECG parameters between gender and donepezil 
doses. We performed point-biserial correlations to assess whether cardiac comorbidities and concurrent use of medications alongside donepezil were associated with changes in ECG parameters. The Kruskal Wallis test was used to evaluate whether any changes in ECG parameters were associated with duration of donepezil treatment. Statistical significance was defined as P-value $<0.05$. Our data and statistical analysis are compliant with the recommendations on experimental design and analysis in pharmacology (Curtis \& Alexander et al., 2018).

Results

We identified 59 patients that were suitable for inclusion in our study. Their baseline demographics and characteristics are summarised in Table 1. Analysis of a resting 12-lead ECG prior to commencing donepezil treatment was compared to the ECG obtained during their most recent acute hospital admission and demonstrated evidence of significant prolongation of the PR, QRS and QT intervals (Table 2). The QT corrected using Bazett, Fredericia, Framingham and Hodges formulae were also compared, and all confirmed significant QT prolongation during donepezil therapy. Analysis using the QT nomogram revealed that 8 (13.6\%) patients (3 male and 5 female) went from low arrhythmogenic risk to high while on donepezil therapy (Figure 1). No significant change in mean HR was noted; although one patient had donepezil ceased on admission due to bradycardia and intermittent complete heart block. Cessation of donepezil resulted in normalisation of this patient's heart rate and resolution of their heart block.

Assessment of the effect of donepezil on ECG parameters demonstrated that males experienced a significantly greater increase in their corrected QT intervals compared to females (Table 3). There were no significant differences in all ECG parameters when comparing donepezil doses (Table 4). Although there was a significant difference in QT interval between the different treatment duration groups, no significant difference was found once QT intervals were corrected. Duration of donepezil therapy did not affect the HR, PR interval or QRS interval (Table 5).

Point-biserial correlation found that concomitant use of rate-limiting calcium channel blockers was associated with significant $\mathrm{PR}$ prolongation $\left(r_{\mathrm{pb}}=0.314, \mathrm{P}=0.030\right)$, while beta-blockers in combination with donepezil were found to significantly reduce the $\mathrm{HR}\left(r_{\mathrm{pb}}=0.256, \mathrm{P}=0.050\right)$. All corrected QT intervals whilst on donepezil were significantly prolonged by the use of tricyclic antidepressants (QTcB: $r_{\mathrm{pb}}=0.344, \mathrm{P}=0.008$, QTcFred: $r_{\mathrm{pb}}=0.382, \mathrm{P}=0.003$, QTcFram: $\left.r_{\mathrm{pb}}=0.379, \mathrm{P}=0.003, \mathrm{QTcH}: r_{\mathrm{pb}}=0.352, \mathrm{P}=0.006\right)$. There was no significant correlation between cardiac comorbidities and changes in ECG parameters.

\section{Discussion and Conclusions}

Our study clearly demonstrated that the use of donepezil for more than a year significantly prolongs the PR, QRS and QT intervals. Male patients had a significantly greater prolongation of their QTc interval compared to females, and concomitant use of tricyclic antidepressants significantly increased the risk of QT interval prolongation, while no dose or treatment duration related differences were found.

The QT interval is an electrocardiographic representation of the time taken for depolarisation and repolarisation of the ventricles. Measuring the QT interval and understanding its significance can be difficult since it is influenced by multiple factors including heart rate and disease-associated ECG changes. The use of corrected QT intervals is more useful in the clinical setting, but the applicability of each formula remains debatable because of over or under correction at extremes of heart rate (Roden, 2008).

A prolonged QT interval has been used as a surrogate marker for predicting subsequent risk of ventricular arrhythmias. However, its significance varies between individuals and is affected by multiple factors including medications, underlying heart rhythm, asystolic pauses and genetics. Furthermore, the relationship between the QT interval and risk of TdP is non-linear. It should therefore be interpreted with caution and not in isolation, with consideration of the aforementioned confounding patient characteristics (Roden, 2008). Despite the above caveats, the risk of ventricular arrhythmias and increased cardiac morbidity and mortality in the presence of a prolonged QT interval are well established. Although its consequences may be unpredictable, QT prolongation does frequently lead to adverse outcomes (Kitt et al., 2015; Mandal et al., 2019; Roden, 
2008; Malik \& Batchvarov, 2000).

Our study confirmed that the use of donepezil for duration of at least 1 year was associated with a significant increase in the QT intervals. Donepezil enhances the cholinergic function of the brain while also causing undesired systemic cholinergic side-effects, with gastrointestinal disturbances such as nausea, vomiting and diarrhoea being the most common. The adverse effects on the heart are unpredictable. The heart is an organ rich in cholinesterase enzymes ( $\mathrm{ChE}$ ). Inhibition of these enzymes leads to increased acetylcholine, which deactivates the membrane bound voltage-gated calcium channels. Phase two of the cardiac action potential is also known as the plateau phase, which is defined by the membrane potential remaining constant due to the concurrent influx of calcium ions and efflux of potassium ions. Donepezil may delay this phase by increasing cardiomyocyte acetylcholine levels, hence deactivating the voltage-gated calcium channels, while simultaneously causing direct inhibition of delayed rectifier potassium channels. This results in QT interval prolongation and a subsequent increased risk of ventricular arrhythmias (Takaya et al., 2009; Malik \& Batchvarov, 2000; Li \& Cheng et al., 2017; Isik \& Bozoglu et al., 2012).

Most studies to date have evaluated ECG changes in patients taking donepezil for up to four months. Significant prolongation of the PR interval is a common finding, with no associated change in the QT interval (Igeta et al., 2014; Wang et al., 2018; Rabkin \& Cheng et al., 2016). We postulate that a longer duration of donepezil therapy may be necessary to demonstrate a significant QT interval prolongation, as found in our study. This finding raises concerns since the adverse effects of QT prolongation and subsequent TdP have only been previously identified in case studies, resulting in the risk being underestimated by clinicians (Kitt et al., 2015; Mandal et al., 2019). Furthermore, it is known that the QT interval increases with age and this may exacerbate the risk of ventricular arrhythmias in an older population (Rabkin et al., 2016). This risk may be further amplified by polypharmacy, electrolyte derangements and concurrent cardiac comorbidities or conduction disorders.

Female gender has been reported in literature to be an independent risk factor for QT prolongation, due to their greater baseline QT interval and oestrogen mediated gender differences in the expression of cardiac delayed rectifier potassium channels (Rabkin, 2015). Interestingly our study demonstrated that male patients experienced a significantly greater prolongation of their corrected QT interval compared to females. While females tend to show a longer QT interval at younger ages, gender differences decrease with age due to males having a more pronounced age-related increase in their QT interval (Rabkin, 2015). This is observed within our cohort with both older males and females having a similar QT interval prior to commencing donepezil therapy (Table 3).

A possible explanation is that male patients have a greater sensitivity to the increase in cardiac acetylcholine levels caused by donepezil, which results in a greater prolongation of the QT interval (Takaya et al., 2009). Naturally there is an increase in vagal tone and acetylcholine release when patients undergo a carotid sinus massage. Male patients exhibit an increased sensitivity to carotid sinus massage and have an increased prevalence of carotid sinus disease, which could theoretically be due to an underlying oversensitivity to acetylcholine (Tan \& Newton et al., 2009; Kumar \& Thomas et al., 2003; Kerr \& Pearce et al., 2006). Further evidence of this is that male patients are less responsive to muscarinic receptor antagonists such as atropine (Tsutsui \& Falcão et al., 2007). This mechanism may explain why male patients exhibit a greater prolongation of the corrected QT interval compared to females, but our results would require confirmation in a larger study.

Another manifestation of the increase in vagal tone caused by donepezil is prolonged PR and QRS intervals, as seen in our study population (Sato \& Urbano et al., 2010). Underlying cardiac conduction disease and the concomitant use of commonly prescribed negatively chronotropic medications could exacerbate this further. We demonstrated that concurrent use of rate limiting calcium channel blockers and beta-blockers in those on donepezil therapy were associated with significant PR prolongation and a reduction in heart rate respectively. One patient in our study population developed symptomatic bradycardia requiring admission. Cessation of donepezil therapy reversed the bradycardia and resulted in normalisation of heart rate. Previous studies have similarly reported hospitalisation for donepezil associated bradycardia, with a proportion of patients 
developing syncope and irreversible heart block requiring permanent pacemaker implantation. Careful consideration must be taken when initiating donepezil in patients already prescribed negatively chronotropic medications (Bordier et al., 2003; Hernandez et al., 2009; Park-Wyllie \& Mamdani et al., 2009).

Our study did not demonstrate a correlation between the duration of donepezil therapy and further QT prolongation (Table 5), but its prolonged use theoretically carries an increased risk of ventricular arrhythmias. The neurocognitive benefits of donepezil are most pronounced in the initial months of treatment and continue for up to two years. Following two years of treatment, the overall benefit of donepezil remains unclear (Courtney \& Farrell et al., 2004). We noted that several of our patients had extended donepezil therapy for longer than the beneficial 2-year treatment period. We therefore suggest that such patients should be reassessed at this point and termination of treatment with donepezil should be considered if the risks of adverse events are deemed to outweigh any clear neurocognitive benefit.

We recognise the limitation of our study as a single centre study in an acute hospital setting with a predominantly Caucasian population, which may limit the generalizability of our results to other hospitals or community settings. We also recognise that ECG recordings took place during acute hospital admissions and the parameters measured may have been affected by confounding factors such as a concurrent acute illness e.g. infection. Our results were based on resting 12-lead ECGs before and during donepezil treatment, and despite providing valuable information they are only a snapshot of the patient's cardiac conduction and may not be representative of their predominant rhythm. A Holter monitor would provide a more reliable assessment of cardiac conduction abnormalities and would also capture intermittent arrhythmias. Therefore a larger prospective study with prolonged cardiac monitoring and longer follow-up period would be of interest.

In conclusion, the results demonstrate that donepezil therapy results in a significant prolongation in the PR and QT intervals. Therefore, we urge clinicians to exercise caution and pharmacovigilance when prescribing donepezil, particularly in patients with pre-existing cardiac comorbidities or among those already prescribed medications known to cause QT prolongation such as tricyclic antidepressants. We suggest a resting 12-lead ECG should take place before and after donepezil initiation and where there is suspicion of QT prolongation, utilisation of a QT nomogram can reliably assess arrhythmogenic risk.

Summary

\section{What is already known}

- Case studies have reported QT prolongation and subsequent Torsades de Pointes associated with donepezil use.

- Cardiac conduction disturbances are considered to be rare side-effects of donepezil.

\section{What this study adds}

Donepezil prolongs QTc intervals, a surrogate marker for predicting subsequent risk of ventricular arrhythmias.

\section{Clinical significance}

ECG evaluation should take place before and after the initiation of donepezil therapy.

\section{References}

Bordier, P., Garrigue, S., Barold, S. S., Bressolles, N., Lanusse, S., \& Clementy, J. (2003). Significance of syncope in patients with alzheimer's disease treated with cholinesterase inhibitors. Europace : European Pacing, Arrhythmias, and Cardiac Electrophysiology: Journal of the Working Groups on Cardiac Pacing, Arrhythmias, and Cardiac Cellular Electrophysiology of the European Society of Cardiology, 5 (4), 429-431. doi:10.1016/s1099-5129(03)00080-1 [doi]

Bordier, P., Garrigue, S., Lanusse, S., Margaine, J., Robert, F., Gencel, L., \& Lafitte, A. (2006). Cardiovascular effects and risk of syncope related to donepezil in patients with alzheimer's disease. CNS Drugs, 20 (5), 411-417. doi:2055 [pii] 
Chan, A., Isbister, G. K., Kirkpatrick, C. M., \& Dufful, S. B. (2007). Drug-induced QT prolongation and torsades de pointes: Evaluation of a QT nomogram. QJM : Monthly Journal of the Association of Physicians, 100 (10), 609-615. doi:hcm072 [pii]

Courtney, C., Farrell, D., Gray, R., Hills, R., Lynch, L., Sellwood, E., . . AD2000 Collaborative Group. (2004). Long-term donepezil treatment in 565 patients with alzheimer's disease (AD2000): Randomised double-blind trial. Lancet (London, England), 363 (9427), 2105-2115. doi:10.1016/S0140-6736(04)16499-4 [doi]

Curtis, M. J., Alexander, S., Cirino, G., Docherty, J. R., George, C. H., Giembycz, M. A., . . Ahluwalia, A. (2018). Experimental design and analysis and their reporting II: Updated and simplified guidance for authors and peer reviewers. British Journal of Pharmacology, 175 (7), 987-993. doi:10.1111/bph.14153

Hernandez, R. K., Farwell, W., Cantor, M. D., \& Lawler, E. V. (2009). Cholinesterase inhibitors and incidence of bradycardia in patients with dementia in the veterans affairs new england healthcare system. Journal of the American Geriatrics Society, 57 (11), 1997-2003. doi:10.1111/j.1532-5415.2009.02488.x [doi]

Igeta, H., Suzuki, Y., Tajiri, M., \& Someya, T. (2014). Cardiovascular pharmacodynamics of donepezil hydrochloride on the PR and QT intervals in patients with dementia. Human Psychopharmacology, 29 (3), 292-294. doi:10.1002/hup.2398 [doi]

Isik, A. T., Bozoglu, E., Yay, A., Soysal, P., \& Ateskan, U. (2012). Which cholinesterase inhibitor is the safest for the heart in elderly patients with alzheimer's disease? American Journal of Alzheimer's Disease and Other Dementias, 27 (3), 171-174. doi:10.1177/1533317512442999 [doi]

Kerr, S. R., Pearce, M. S., Brayne, C., Davis, R. J., \& Kenny, R. A. (2006). Carotid sinus hypersensitivity in asymptomatic older persons: Implications for diagnosis of syncope and falls. Archives of Internal Medicine, 166 (5), 515-520. doi:166/5/515 [pii]

Kumar, N. P., Thomas, A., Mudd, P., Morris, R. O., \& Masud, T. (2003). The usefulness of carotid sinus massage in different patient groups. Age and Ageing, 32 (6), 666-669. doi:10.1093/ageing/afg114 [doi]

Li, K., Cheng, N., \& Li, X. T. (2017). Inhibitory effects of cholinesterase inhibitor donepezil on the Kv1.5 potassium channel. Scientific Reports, 7 , 41509. doi:10.1038/srep41509 [doi]

Malik, B. H., Hamid, P., Khan, S., Gupta, D., \& Islam, M. (2019). Correlation between donepezil and QTc prolongation and torsades de pointes: A very rare phenomenon. Cureus, 11 (12), e6451. doi:10.7759/cureus.6451 [doi]

Malik, M., \& Batchvarov, V. N. (2000). Measurement, interpretation and clinical potential of QT dispersion. Journal of the American College of Cardiology, 36 (6), 1749-1766. doi:S0735-1097(00)00962-1 [pii]

Mandal, A. K. J., Khan, U. F., \& Missouris, C. G. (2019). Ventricular tachycardia in a patient with sigmoid volvulus on treatment with donepezil. Clinical Toxicology (Philadelphia, Pa.), 57 (12), 1157-1159. doi:10.1080/15563650.2019.1588288 [doi]

Park-Wyllie, L. Y., Mamdani, M. M., Li, P., Gill, S. S., Laupacis, A., \& Juurlink, D. N. (2009). Cholinesterase inhibitors and hospitalization for bradycardia: A population-based study. PLoS Medicine, 6 (9), e1000157. doi:10.1371/journal.pmed.1000157 [doi]

Rabkin, S. W. (2015). Impact of age and sex on QT prolongation in patients receiving psychotropics. Canadian Journal of Psychiatry.Revue Canadienne De Psychiatrie, 60 (5), 206-214. doi:cjp-2015-vol60-may-206-214 [pii]

Rabkin, S. W., Cheng, X. J., \& Thompson, D. J. (2016). Detailed analysis of the impact of age on the QT interval. Journal of Geriatric Cardiology : JGC, 13 (9), 740-748. doi:jgc-13-09-740 [pii]

Roden, D. M. (2008). Keep the QT interval: It is a reliable predictor of ventricular arrhythmias. Heart Rhythm, 5 (8), 1213-1215. doi:10.1016/j.hrthm.2008.05.008 [doi] 
Sato, K., Urbano, R., Yu, C., Yamasaki, F., Sato, T., Jordan, J., . . . Diedrich, A. (2010). The effect of donepezil treatment on cardiovascular mortality. Clinical Pharmacology and Therapeutics, 88 (3), 335-338. doi:10.1038/clpt.2010.98 [doi]

Takaya, T., Okamoto, M., Yodoi, K., Hata, K., Kijima, Y., Nakajima, H., . . . Kawashima, S. (2009). Torsades de pointes with QT prolongation related to donepezil use. Journal of Cardiology, 54 (3), 507-511. doi:10.1016/j.jjcc.2009.03.011 [doi]

Tan, M. P., Newton, J. L., Reeve, P., Murray, A., Chadwick, T. J., \& Parry, S. W. (2009). Results of carotid sinus massage in a tertiary referral unit-is carotid sinus syndrome still relevant? Age and Ageing, 38 (6), 680-686. doi:10.1093/ageing/afp160 [doi]

Tsutsui, J. M., Falcao, S. N., Dourado, P. M., Lima, M. F., Alves, A. A., Guerra, V. C., . . Mathias, W. (2007). Gender differences in chronotropic and hemodynamic responses during dobutamine-atropine stress echocardiography. Echocardiography (Mount Kisco, N.Y.), 24 (8), 843-850. doi:ECHO487 [pii]

United States Food and Drug Administration. (2012). Aricept (donepezil hydrochloride). Retrieved from https://www.accessdata.fda.gov/drugsatfda_docs/label/2012/020690s035,021720s008,022568s005lbl.pdf

Wang, D., Wu, Y., Wang, A., Chen, Y., Zhang, T., \& Hu, N. (2018). Electrocardiogram changes of donepezil administration in elderly patients with ischemic heart disease. Cardiology Research and Practice, 2018 , 9141320. doi:10.1155/2018/9141320 [doi] 
Figure 1 A comparison of QT nomogram plots before (top) and after (bottom) the initiation of donepezil therapy.
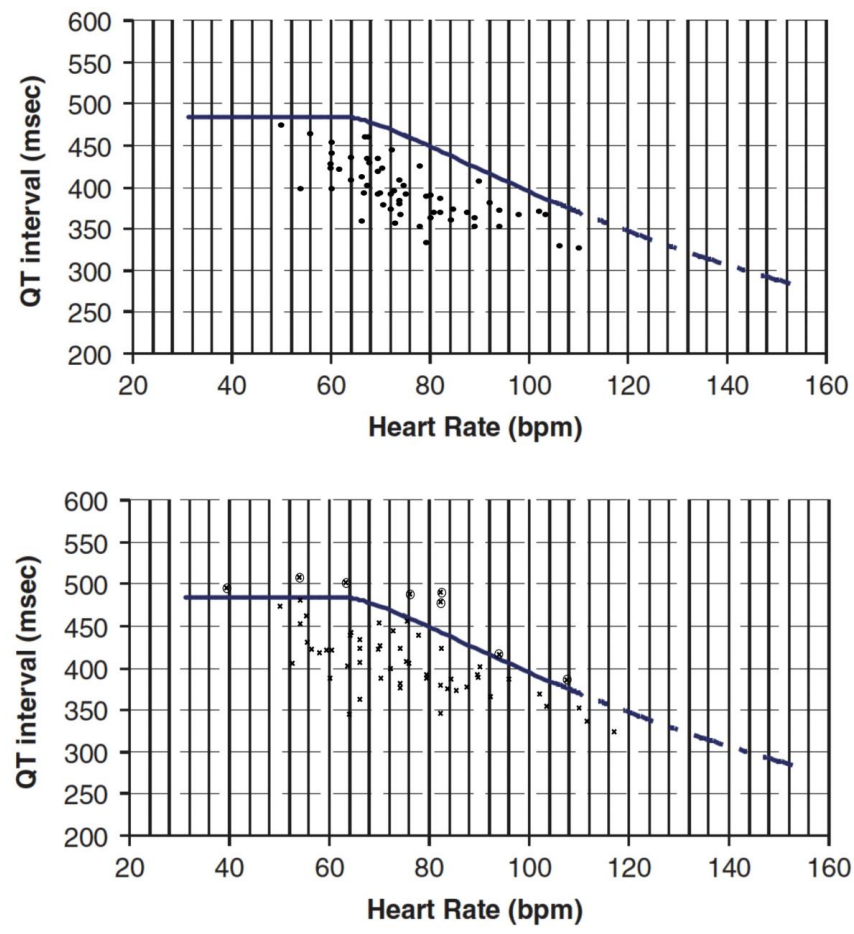
Table 1 Patient Demographics and Characteristics

\begin{tabular}{|c|c|}
\hline Patient Characteristics & $\mathbf{N}(\%)$ \\
\hline Total & 59 \\
\hline Age (years) & $86 \pm 5$ \\
\hline Female & $37(63 \%)$ \\
\hline \multicolumn{2}{|l|}{ Ethnicity } \\
\hline Caucasian & $50(85 \%)$ \\
\hline Others & $9(15 \%)$ \\
\hline \multicolumn{2}{|l|}{ Donepezil dose } \\
\hline $5 \mathrm{mg}$ & $25(42 \%)$ \\
\hline $10 \mathrm{mg}$ & $34(58 \%)$ \\
\hline \multicolumn{2}{|l|}{ Indication } \\
\hline Alzheimer's dementia & $29(49.2 \%)$ \\
\hline Unspecified dementia & $25(42.4 \%)$ \\
\hline Vascular dementia & $3(5.1 \%)$ \\
\hline Mixed dementia & $2(3.4 \%)$ \\
\hline \multicolumn{2}{|l|}{ Reason for admission } \\
\hline Fall & 27 \\
\hline Confusion/Delirium & 7 \\
\hline Infection & 27 \\
\hline \multicolumn{2}{|l|}{ Duration on Donepezil (nearest year) } \\
\hline 1 year & 20 \\
\hline 2 years & 5 \\
\hline 3 years & 10 \\
\hline 4 years & 5 \\
\hline 5 years & 6 \\
\hline \multicolumn{2}{|l|}{ Serum biochemistry } \\
\hline Potassium (mmol/L) & $4.43 \pm 0.53$ \\
\hline Magnesium (mmol/L) & $0.80 \pm 0.17$ \\
\hline Adjusted Calcium (mmol/L) & $2.42 \pm 0.12$ \\
\hline Thyroid-Stimulating Hormone (mIU/L) & $2.27 \pm 2.60$ \\
\hline \multicolumn{2}{|l|}{ Comorbidities } \\
\hline Hypertension & 42 \\
\hline
\end{tabular}


Table 2 Comparison of the ECG parameters before donepezil initiation and once patients were on donepezil treatment.

\begin{tabular}{lcccc}
\hline ECG Parameters & Baseline & On Donepezil & $\Delta$ & P-value \\
\hline HR (beats/min) & $76.2 \pm 13.7$ & $75.5 \pm 17.1$ & & 0.513 \\
PR interval (ms) & $177.0 \pm 29.0$ & $186.1 \pm 34.2$ & 9.1 & $0.042^{*}$ \\
QRS duration (ms) & $101.7 \pm 20.3$ & $104.7 \pm 22.3$ & 3.0 & $0.040^{*}$ \\
QT interval (ms) & $393.3 \pm 35.6$ & $411.9 \pm 44.6$ & 18.6 & $0.002^{*}$ \\
QTcBazett (ms) & $438.9 \pm 28.7$ & $455.7 \pm 43.4$ & 16.8 & $0.009^{*}$ \\
QTcFred (ms) & $422.7 \pm 26.2$ & $439.6 \pm 37.4$ & 16.9 & $0.001^{*}$ \\
QTcFram (ms) & $422.2 \pm 24.7$ & $437.1 \pm 35.4$ & 14.9 & $0.003^{*}$ \\
& & & & \\
QTcHodges (ms) & $421.7 \pm 25.2$ & $439.2 \pm 34.7$ & 17.5 & $0.001^{*}$ \\
& & & & \\
\hline
\end{tabular}


Table 3 Comparison of how ECG parameters are affected by gender while on treatment with donepezil

\begin{tabular}{lccccccc}
\hline ECG Parameters & \multicolumn{3}{c}{ Male } & \multicolumn{3}{c}{ Female } & P-value \\
\cline { 2 - 5 } & Baseline & On Donepezil & $\Delta$ & Baseline & On Donepezil & $\Delta$ & \\
\hline HR (beats/min) & $75.1 \pm 15.6$ & $76.4 \pm 20.4$ & 1.3 & $76.8 \pm 12.7$ & $75.0 \pm 15.0$ & 1.8 & 0.638 \\
PR interval (ms) & $178.1 \pm 21.1$ & $188.1 \pm 30.9$ & 10.0 & $176.5 \pm 32.7$ & $185.1 \pm 36.2$ & 8.6 & 0.294 \\
QRS duration (ms) & $107.5 \pm 22.6$ & $107.0 \pm 23.3$ & 0.5 & $98.3 \pm 18.3$ & $103.3 \pm 21.9$ & 5.0 & 0.106 \\
QT interval (ms) & $398.4 \pm 40.2$ & $424.2 \pm 51.0$ & 25.8 & $390.3 \pm 32.9$ & $404.6 \pm 39.2$ & 14.3 & 0.060 \\
QTcBazett (ms) & $440.3 \pm 32.0$ & $468.5 \pm 30.3$ & 28.2 & $438.0 \pm 27.0$ & $448.1 \pm 48.4$ & 10.1 & $0.023^{*}$ \\
QTcFred (ms) & $425.4 \pm 28.5$ & $451.5 \pm 30.6$ & 26.1 & $421.1 \pm 25.0$ & $432.6 \pm 39.5$ & 11.5 & $0.049^{*}$ \\
QTcFram (ms) & $424.4 \pm 27.0$ & $449.5 \pm 30.4$ & 25.1 & $421.0 \pm 23.5$ & $429.8 \pm 36.5$ & 8.8 & $0.021^{*}$ \\
QTcHodges (ms) & $425.0 \pm 28.3$ & $452.9 \pm 26.6$ & 27.9 & $419.7 \pm 23.4$ & $431.0 \pm 36.7$ & 11.3 & $0.015^{*}$ \\
\hline
\end{tabular}

Table 4 Comparison of how ECG parameters are affected by the dose of donepezil

\begin{tabular}{lccccccc}
\hline ECG Parameters & \multicolumn{3}{c}{ 5mg } & & & 10mg & P-value \\
& \multicolumn{1}{c}{ Baseline } & On Donepezil & $\Delta$ & Baseline & On Donepezil & $\Delta$ & \\
\hline HR (beats/min) & $79.2 \pm 14.2$ & $75.0 \pm 19.7$ & 4.2 & $73.9 \pm 13.1$ & $75.9 \pm 15.1$ & 2.0 & 0.074 \\
PR interval (ms) & $178.6 \pm 31.9$ & $186.9 \pm 34.8$ & 8.3 & $175.9 \pm 27.1$ & $185.6 \pm 34.4$ & 9.7 & 0.819 \\
QRS duration (ms) & $98.9 \pm 20.6$ & $100.6 \pm 21.0$ & 1.7 & $103.7 \pm 20.1$ & $107.6 \pm 23.0$ & 3.9 & 0.452 \\
QT interval (ms) & $382.2 \pm 36.0$ & $411.7 \pm 45.3$ & 29.5 & $401.5 \pm 33.6$ & $412.0 \pm 44.8$ & 10.5 & 0.158 \\
QTcBazett (ms) & $435.0 \pm 26.5$ & $451.8 \pm 43.0$ & 16.8 & $441.7 \pm 30.3$ & $458.6 \pm 44.1$ & 16.9 & 0.565 \\
QTcFred (ms) & $416.1 \pm 25.0$ & $437.3 \pm 34.4$ & 21.2 & $427.6 \pm 26.4$ & $441.3 \pm 39.8$ & 13.7 & 0.824 \\
QTcFram (ms) & $416.1 \pm 23.0$ & $433.8 \pm 32.2$ & 17.7 & $426.7 \pm 25.3$ & $439.5 \pm 37.9$ & 12.8 & 0.988 \\
QTcHodges (ms) & $415.9 \pm 23.8$ & $438.0 \pm 32.4$ & 22.1 & $425.9 \pm 25.7$ & $440.0 \pm 36.8$ & 14.1 & 0.736 \\
\hline
\end{tabular}




\begin{tabular}{|c|c|c|c|c|c|c|}
\hline \multirow[t]{2}{*}{ ECG Parameters } & \multicolumn{5}{|c|}{ Duration (years) } & \multirow[t]{2}{*}{ P-value } \\
\hline & 1 & 2 & 3 & 4 & 5 & \\
\hline$\Delta$ Heart Rate $(\mathrm{bpm})$ & $-4.3 \pm 16.4$ & $15.6 \pm 23.4$ & $-1.3 \pm 15.1$ & $24.0 \pm 34.7$ & $-10.2 \pm 21.3$ & 0.159 \\
\hline$\Delta \mathrm{PR}$ Interval (ms) & $6.53 \pm 31.0$ & $49.0 \pm 59.4$ & $13.1 \pm 27.2$ & $-3.0 \pm 24.9$ & $17.3 \pm 30.8$ & 0.595 \\
\hline$\Delta \mathrm{QRS}$ Interval (ms) & $-1.6 \pm 12.4$ & $6.5 \pm 15.9$ & $10.5 \pm 14.9$ & $0.4 \pm 5.7$ & $5.0 \pm 5.0$ & 0.302 \\
\hline$\Delta \mathrm{QT}$ Interval (ms) & $27.6 \pm 46.1$ & $5.0 \pm 61.8$ & $12.3 \pm 31.4$ & $-50.6 \pm 68.8$ & $64.3 \pm 24.2$ & $0.012 *$ \\
\hline$\triangle \mathrm{QTcB}$ Interval (ms) & $14.5 \pm 58.9$ & $51.4 \pm 34.6$ & $8.1 \pm 35.6$ & $9.2 \pm 21.8$ & $40.0 \pm 61.0$ & 0.201 \\
\hline$\Delta$ QTcFred Interval (ms) & $18.8 \pm 50.2$ & $34.6 \pm 35.9$ & $9.3 \pm 23.4$ & $-12.6 \pm 15.9$ & $45.3 \pm 39.3$ & 0.051 \\
\hline$\Delta$ QTcFram Interval (ms) & $16.4 \pm 45.9$ & $28.6 \pm 35.4$ & $6.7 \pm 24.6$ & $-13.6 \pm 17.9$ & $46.8 \pm 33.8$ & 0.052 \\
\hline$\Delta \mathrm{QTcH}$ Interval (ms) & $20.2 \pm 47.6$ & $32.6 \pm 32.4$ & $10.0 \pm 19.1$ & $-8.4 \pm 16.3$ & $46.7 \pm 36.9$ & 0.080 \\
\hline
\end{tabular}

